\title{
Pós-graduação na educação física brasileira: a atração (fatal) para a biodinâmica
}

Edison de Jesus Manoel

Yara Maria de Carvalho

Universidade de São Paulo

\section{Resumo}

0 objetivo do trabalho foi caracterizar academicamente a educação física no Brasil. Primeiro, fez-se um paralelo entre os eventos desse processo ocorridos nos Estados Unidos e no Brasil. A seguir, os programas de pós-graduação brasileiros foram analisados do ponto de vista de suas áreas de concentração e de sua vinculação com o corpo docente, as linhas e os projetos de pesquisa. Educação física é o termo predominante na denominação da maioria dos programas brasileiros, diferentemente dos Estados Unidos, onde se privilegia cinesiologia. A análise das áreas de concentração dos programas de pós-graduação permitiu-nos identificar três subáreas: biodinâmica, sociocultural e pedagógica. A biodinâmica sobressai-se pela dimensão do corpo docente e pela quantidade de linhas e projetos de pesquisa, sempre mais numerosos em comparação com as subáreas sociocultural e pedagógica. Tal hegemonia expressa a valorização atribuída às pesquisas orientadas pelas ciências naturais em detrimento daquelas fundamentadas pelas ciências humanas e sociais, além da dificuldade em problematizar a intervenção, particularmente no âmbito da escola. Esse quadro guarda semelhança com a realidade norteamericana, haja vista que acadêmicos norte-americanos das subáreas sociocultural e pedagógica apontam os obstáculos para compatibilizar suas concepções teóricas e metodológicas com os modos hegemônicos de pensamento, investigação e ação no campo da cinesiologia. Tanto os acontecimentos nos Estados Unidos quanto a caracterização acadêmica predominante nos programas de pós-graduação no Brasil indicam a forte presença da biodinâmica em prejuízo da educação física, no que ela compreende e agrega, sobretudo, como prática social e pedagógica de longa data.

\section{Palavras-chave}

Educação física - Cinesiologia - Biodinâmica - Política científica Pós-graduação 


\title{
Graduate studies in Brazilian physical education: the (fa- tal) attraction to biodynamics
}

\author{
Edison de Jesus Manoel \\ Yara Maria de Carvalho \\ Universidade de São Paulo
}

Contact:

Edison de Jesus Manoel Rua Paraguai, 300

06350-170 - Carapicuiba /SP

E-mail: ejmanoel@usp.br

\begin{abstract}
The present work aimed at the academic characterization of physical education in Brazil. First, a parallel was made between the history of academic characterization of physical education in North America and in Brazil. Next, the analysis of the areas comprehended by graduate study programs was carried out in the field around Brazil. A survey was done considering the field of concentration and its interface and links with the size of faculty, with research lines and with research projects. Physical education is the most preferred term to name the majority of the Brazilian graduate programs in contrast with the United States where Kinesiology is preferred. The analysis of the field of concentration yields three main subfields: biodynamics, sociocultural and pedagogical. Biodynamics takes precedence as one considers the size of the faculty and the number of research lines and projects always greater than the same variables in comparison with sociocultural and pedagogical subfields. This hegemony reflects a trend in which natural sciences-oriented research is privileged over human and social sciences-oriented research and difficulty in valuing the intervention, especially in schooling. This portrait resembles what happens in the US as some North American scholars from the sociocultural and pedagogical subfields have also identified difficulties in making their theoretical and methodological conceptions compatible with the hegemonic modes of thinking and investigation in kinesiology.
\end{abstract}

\section{Keywords}

Physical education - Kinesiology - Biodynamics - Scientific policy - Graduate studies 
A educação física tem diversas finalidades e associações (com a educação, a saúde, o esporte, o lazer); ainda assim ela é pensada como sendo pedagógica em essência (BRACHT, 2003, 2007). Como campo de conhecimento, a educação física é relativamente jovem e as dificuldades para sua afirmação passam pela discussão relativa a seu objeto de estudo, suas afınidades com as ciências naturais e com as humanas e sociais, sua legitimidade no âmbito acadêmico-científico, seu reconhecimento como ciência ou como prática social e seu papel no ensino superior. 0 objetivo do presente artigo é caracterizar academicamente a educação física a partir da orientação dos programas de pós-graduação da área. A tese é a de que a educação física é negligenciada como prática social e campo de conhecimento que instiga o diálogo entre saberes e práticas diversas, sejam eles da educação, das ciências da saúde, das ciências biológicas ou das ciências sociais e humanas.

\section{A caracterização acadêmica da educação física brasileira}

A constituição acadêmica da educação física brasileira seguiu um caminho diferente em comparação ao que ocorreu na América do Norte. No início dos anos 1960, as universidades norte-americanas estavam sob escrutínio avaliativo e o status acadêmico de muitos departamentos - entre eles, os de educação física - estava sendo questionado. Na época, Franklin Henry, chefe do Departamento de Educação Física da Universidade da Califórnia, fez o famoso discurso em defesa da educação física como uma disciplina acadêmica legítima, argumentando que os conhecimentos ensinados nos cursos de graduação da área eram produzidos por ela (HENRY, 1964). Henry falou sobre como a educação física era então conduzida na Universidade da Califórnia (PARK, 1994) e argumentou que ela deveria ser vista como uma ciência com objeto de estudo próprio o movimento humano - e com métodos de pesquisa adaptados de campos tradicionais como a biologia, a psicologia, a educação e a sociologia. Lawrence Rarick (1967) avançou ao caracterizar as subdisciplinas da disciplina acadêmica: fisiologia do exercício; crescimento e desenvolvimento motor; controle e aprendizagem motora; psicologia do esporte; sociologia do esporte; história do esporte; entre outras. Assim foi desencadeado o chamado movimento disciplinar: a gradual transformação dos departamentos de educação física em departamentos de cinesiologia e ciência do exercício (NEWELL, 1990).

No Brasil, a caracterização acadêmica da educação física foi, de certa forma, influenciada pelo que ocorreu na América do Norte $^{1}$. 0 início desse processo se deu pela criação de programas de pós-graduação na área $^{2}$ entre o final dos anos 1970 e o início dos anos 1980. Todavia, só a Universidade Federal de Santa Maria mostrava-se sob influência norte-americana ao nomear seu programa de Ciência do Movimento Humano, organizado com base em um conjunto de subdisciplinas (biomecânica, fisiologia do exercício, aprendizagem motora, crescimento e desenvolvimento, etc.). Nos outros programas, adotou-se o termo educação física, e seus cursos eram extensões de disciplinas tradicionais de áreas como educação e biologia.

Desde 1990, o sistema de recomendação estabelecido pela CAPES $^{3}$ começou a valorizar o grau de caracterização acadêmica dos programas, considerando a clareza e

1- Entre os anos 1970 e 1980, um grupo de profissionais de educação física trabalhando em universidades públicas estaduais e federais recebeu bolsas de estudo do governo brasileiro para realizar programas de pós-graduação em nível de mestrado e doutorado em universidades norte-americanas.

2- As universidades que ofereceram programas em nível de mestrado foram: Universidade de São Paulo, Universidade Federal do Rio de Janeiro e Universidade Federal de Santa Maria.

3- CAPES é a sigla que denomina a Coordenação de Aperfeiçoamento de Pessoal de Ensino Superior, uma divisão do Ministério da Educação com a missão de estabelecer políticas e orientações para o sistema brasileiro de educação superior em nível de pós-graduação. 
a consistência na definição da área básica, das linhas de pesquisa, dos projetos de pesquisa e das atividades discentes (seminários, cursos, disciplinas). As diferenças a esse respeito eram grandes entre os programas e frequentemente eram interpretadas como falta de identidade da área. Iniciou-se, então, uma discussão sobre a caracterização acadêmica da área, o que foi expresso em algumas publicações. 0 periódico Motus Corporis ${ }^{4}$, por exemplo, convidou três acadêmicos para escreverem sobre suas visões acerca da educação física. Para Hugo Lovisolo (1996), a educação física não era uma disciplina acadêmica; ao contrário, ela deveria ser vista como uma arte de ensinar que se beneficia de vários tipos de conhecimento, científicos e não científicos. Contrapondo-se a essa visão, Go Tani (1996) defendeu que a educação física era uma disciplina acadêmica que investigava o movimento humano, devendo ser renomeada para cinesiologia, como proposto por Karl Newell (1990). Mauro Betti (1996) colocou-se em uma posição intermediária ao defender a educação física como um campo de intervenção pedagógica, mas que deveria, ao mesmo tempo, desenvolver uma teoria de sua prática com sólidas bases científicas.

Outras publicações expandiram o debate, ainda que na maioria dos casos houvesse uma controvérsia considerável sobre transformar a educação física em ciência do movimento. Por exemplo, o periódico Movimento ${ }^{5}$ lançou a questão "O que é educação física?" para que vários docentes expressassem suas visões nem sempre consensuais sobre as bases epistemológicas da área (entre outros, GAYA, 1994; TAFFAREL; ESCOBAR, 1994; BRACHT, 1995; LOVISOLO, 1995; SANTIN, 1995).

4- Motus Corporis foi um periódico dedicado a publicar trabalhos produzidos dentro programa de pós-graduação da Universidade Gama Filho, do Rio de Janeiro.

5- Movimento é um dos periódicos mais importantes da educação física brasileira, porque sua política editorial privilegia artigos e ensaios que se fundamentam nas ciências humanas e sociais, possibilitando aos pesquisadores das subáreas sociocultural e pedagógica veicularem resultados de pesquisas e reflexões. Para conhecer seu percurso, recomendamos o site <http://seer.ufrgs.br/Movimento>.
Valter Bracht (2003) destacou o fascínio que a visão científica da educação física exercia sobre a comunidade como responsável pelo distanciamento do campo em relação à intervenção pedagógica. Bracht argumentou que, ao assumir a retórica da ciência, a educação física converteu-se a um modo hegemônico de fazer ciência (estritamente falando, o da ciência natural), distanciando-se da pesquisa e da prática pedagógica.

Ao longo dos anos 1990, ocorreu uma mudança gradual na estruturação dos programas de pós-graduação. 0 termo educação física foi mantido, mas houve a proposição de diferentes áreas de concentração com inspiração no movimento disciplinar. Cada área de concentração correspondeu a uma subárea, sendo identificadas três: biodinâmica, sociocultural e pedagógica. A biodinâmica compreende as atividades de pesquisa dentro de subdisciplinas como bioquímica do exercício, biomecânica, fisiologia do exercício, controle motor, aprendizagem e desenvolvimento motor, além de alguns campos aplicados, como nutrição esportiva e treinamento físico e desportivo. As linhas de pesquisa na biodinâmica são orientadas pelas ciências naturais (ABERNETHY, 1996; AMADIO; BARBANTI, 2000). A subárea sociocultural trata de temas como esporte, práticas corporais e atividade física nas perspectivas da sociologia, da antropologia, da história e da filosofia. A subárea pedagógica investiga questões relativas à formação de professores, ao desenvolvimento curricular, aos métodos de ensino e à pedagogia do esporte, além de tratar de aspectos metodológicos, sociais, políticos e filosóficos da educação. As subáreas sociocultural e pedagógica definem suas linhas de investigação orientadas pelas ciências sociais e humanas. Nesse sentido, a educação física investiga em estreita proximidade com a área da educação (BAIN, 1995; BRACHT, 2006), com a sociologia (BETTI, 2009), com a filosofia (FENSTERSEIFER, 1996; KRETCHEMAR, 1994) e com a história (SOARES, 1998). 
Em 1998, a avaliação da pós-graduação passou a ter como referência a internacionalização. 0 propósito era identificar o estágio do programa nesse processo. Passou-se a quantificar o número de artigos em periódicos com alto fator de impacto publicados por docentes e discentes dos programas (FERREIRA; MOREIRA, 2002). Artigos científicos tornaram-se os itens mais valorizados na avaliação, sendo classificados de acordo com os periódicos em que foram publicados, os quais, por sua vez, eram julgados com base em indicadores elaborados pelo Institute of Scientific Information (ISI). Esse instituto gera, para cada periódico, um fator de impacto composto de vários elementos: da quantidade de vezes que uma revista é citada por outras, ao número e idade das referências bibliográficas contidas nos artigos de um dado periódico (GARFIELD, 1994). Também foram consideradas outras bases, como MEDLINE, ERIC, Psychinfo, SciELO e LILACS. Entretanto, a falta de um fator de impacto aceito amplamente fez com que periódicos indexados nessas bases fossem menos valorizados em comparação àqueles que apareciam no Journal of Citation Reports, publicado pelo ISI. Assim, a produção dos docentes do programa era qualificada indiretamente, já que o critério adotado referia-se à indexação de um dado periódico e não à qualidade de artigos produzidos.

0 uso desse critério para avaliar a qualidade da produção científica recebeu muitas críticas. Eugene Garfield (1983), mentor dos indicadores bibliométricos, argumentou que os fatores de impacto dos periódicos e a razão de citações são números que têm significado para cientistas da informação e que sua simples aplicação para outros propósitos, como avaliação acadêmica de um docente ou avaliação da qualidade da produção intelectual de um programa, pode gerar julgamentos equivocados. Há diferenças expressivas nos fatores de impacto de periódicos de áreas diferentes que não implicam diferenças de qualidade entre eles. Algumas citações devem ser contextualizadas, tais como autocitações (um autor que cita seu próprio trabalho, ou citações feitas por pesquisadores que colaboram entre si) e citações feitas sem que haja qualquer apreciação do conteúdo do artigo citado. 0 uso indiscriminado desses fatores de impacto pode gerar distorções na avaliação de muitas áreas, geralmente naquelas relacionadas às ciências sociais e humanas; na educação física, não é diferente (CARVALHO; MANOEL, 2006; RODRIGUES, 2007).

Amanda Machado, Orlando Lourenço e Francisco Silva (2000) citam a psicologia para chamar a atenção para o perigo da busca desenfreada por produtividade. Segundo eles, o aumento significativo no número de artigos publicados a cada ano (aproximadamente 10.000) não corresponde a avanços teóricos importantes no campo. A maioria dos artigos originais relata dados empíricos que apenas replicam resultados já conhecidos, utilizando diferentes amostras e instrumentos. Os laboratórios de pesquisa assemelham-se a linhas de montagem, expondo a fragmentação da área. Investigações de natureza teórico-conceitual têm pouco espaço para veiculação, sobretudo devido às políticas editoriais dos periódicos, as quais estimulam a produção de pesquisas originais que, no final, resultam em um número muito maior de artigos.

A política de avaliação instalou um processo de indução no qual a caracterização acadêmica da educação física é governada por temas de pesquisa que apresentam maior probabilidade de serem publicados em periódicos com alto fator de impacto, independentemente de esses temas serem significativos ou pertinentes ao campo. Em decorrência, o crescimento da área ou de algumas subáreas vê-se restringido ao número de periódicos indexados no ISI, número este que é maior para as áreas de ciências naturais do que para as de ciências humanas e sociais (GARFIELD, 1994). Uma relação similar é encontrada entre periódicos associados à educação física no ISI. A maioria dos periódicos 
indexados no ISI é de orientação biológica, em detrimento daqueles de orientação sociocultural (HOPKINS, 2001). 0 maior número de periódicos nas ciências naturais faz com que seus fatores de impacto sejam maiores em relação aos das ciências sociais e humanas (WATERS, 2006); na educação física, também não é diferente (CARVALHO; MANOEL, 2006; RODRIGUES, 2007).

A avaliação é fundamental no processo de construção de um campo, além de estabelecer um terreno comum entre diferentes áreas. Entretanto, dependendo da forma como ela é administrada, pode exercer, e de fato exerce, um poder normativo e coercitivo. No caso da educação física brasileira, a avaliação tem privilegiado a produção biologicamente orientada em detrimento da produção de outras áreas, humanas e sociais. Pesquisas de orientação biológica têm mais chance de serem publicadas em periódicos com alto fator de impacto, melhorando as condições das subáreas fundamentadas nas ciências naturais.

A maioria dos programas de pós-graduação em educação física oferece apenas titulação em nível de mestrado. Programas que oferecem títulos de doutor são recentes e poucos. 0 primeiro programa em nível de doutorado no Brasil começou em 1989, e, até o momento, apenas nove instituições de ensino superior têm a recomendação para outorgarem esse título, o que é insuficiente dado o número de mestres formados e a necessidade por recursos humanos mais qualificados para conduzir pesquisas e o ensino nas universidades (KOKUBUN, 2003; LOVISOLO, 2005). Com poucas mudanças em vista, aqueles que almejam o doutorado em educação física devem escolher entre dois caminhos: buscar o título de doutor em programas orientados por ciências biológicas e médicas no Brasil ou no exterior, ou procurar programas de pós-graduação em educação, filosofia, antropologia e história. A falta de equilíbrio entre o número de programas de pós-graduação que oferecem títulos de doutor e o número de profissionais que desejam tais títulos tem um efeito duplo para uma área acadêmica ainda jovem como é a educação física. Os membros do corpo docente que obtêm seus doutorados em outras áreas adquiriram, em tese, uma fundamentação sólida em campos de tradição acadêmica, o que pode contribuir para a consolidação da educação física. Todavia, a experiência com pesquisa em outras áreas tem feito com que muitos docentes acabem se engajando com uma agenda de pesquisa que nem sempre é relevante para a área. Aqueles que retornam ao Brasil depois de anos fazendo doutorado no exterior começam a conduzir pesquisas sem levar em consideração as necessárias adaptações à realidade brasileira (DANTAS, 2004).

No geral, há uma distorção nas formas diversas de valorizar as diferentes atividades de pesquisa, isto é, privilegiando algumas em detrimento de outras. 0 impacto desse processo pode ser apreciado pelo levantamento dos programas de pós-graduação no Brasil.

\section{Os programas de pós-graduação em educação física no Brasil:}

uma análise demográfica

Vinte e um programas analisados foram recomendados ${ }^{6}$ pela CAPES até outubro de 2009 (tabela 1). A maioria deles situa-se nas regiões Sul (seis programas) e Sudeste (10 programas) do Brasil; as exceções são dois programas no Centro-Oeste e um no Nordeste do país ${ }^{7}$. Para cada programa é disponibilizado um conjunto de documentos concernentes à sua missão, sua proposta geral, suas linhas e projetos de pesquisa, seu corpo docente, bem

6- Desde que escrevemos este artigo, a CAPES recomendou mais um programa (Universidade São Judas Tadeu) para oferecer título de doutor em educação física. Essa adição não afetou o quadro geral do campo que descrevemos aqui.

7- Dados disponíveis no sítio da CAPES (<http://www.capes.gov.br/avaliaca0>), em relação à grande área da saúde. 
como um sumário de sua formação, suas atividades de pesquisa, seu ensino e orientação e o currículo de disciplinas. Esses documentos são apresentados para cada ano de um dado período. As informações levantadas são de 2006 pelo fato de serem as informações mais fidedignas, uma vez que esse foi o ano do último levantamento nacional (período 2004/2006) realizado pela CAPES $^{8}$. Houve quatro exceções, com programas recomendados recentemente e que ainda não tinham seus dados disponibilizados no sítio da CAPES. Nesses casos, as informações foram obtidas diretamente de seus sítios virtuais em outubro de 2009.

A avaliação dos programas de pós-graduação em educação física é fortemente orientada pelo número de artigos publicados em periódicos com fator de impacto (indexados em uma das bases ISI) dividido pelo número de docentes do programa. A produção publicada em periódicos brasileiros é desvalorizada independentemente de seu impacto científico e de sua importância social, considerando as necessidades nacionais, regionais e locais. Livros são também desvalorizados; entretanto, desde 2005, há uma comissão trabalhando com a tarefa de estabelecer os parâmetros para avaliação da produção em livro junto aos programas de pós-graduação (CARVALHO; MANOEL, 2007; CARVALHO et al., 2008). Em que pesem os avanços na avaliação da produção intelectual em livros, a produção de artigos ainda é o item mais valorizado na avaliação dos programas. Esse procedimento causa grandes distorções para a avaliação da produção intelectual, considerando que os conceitos de 1 a 7 são atribuídos com base nele. Tal escala reflete a

8- A CAPES finalizou recentemente o levantamento correspondente aos anos 2007-2009, mas os dados e documentos não estarão disponíveis para livre acesso até 0 final do ano 2010. política científica referente à pós-graduação no Brasil, que privilegia e instiga instituições universitárias a almejarem os conceitos 6 e 7. Isso seria lógico se os critérios aplicados nos procedimentos de avaliação não tivessem um viés no qual artigos em periódicos indexados no ISI recebem mais pontos. Assim, pesquisas nas ciências físicas e biológicas são mais valorizadas.

As instituições com programas na área são possuídas por uma real obsessão em se tornarem internacionais, isto é, em obterem os conceitos 6 e 7, às custas de darem as costas à pesquisa nas subáreas sociocultural e pedagógica. Em geral, essas atividades de pesquisa têm impacto local, regional e nacional e, por isso, podem não ser do interesse de periódicos internacionais. A preocupação dos pesquisadores envolvidos nesse tipo de estudo é fornecer respostas aos dilemas que se colocam perante a educação e a saúde brasileira; dessa forma, estabelecem um diálogo com colegas que vivem as mesmas realidades cultural, social, política e econômica. Internacionalização é um fenômeno social que afeta a educação de várias formas, mas envolve bem mais do que publicar artigos em periódicos internacionais (NOGUEIRA; AGUIAR; RAMOS, 2008). 0 impacto da política de internacionalização torna-se evidente na maneira como os programas de pós-graduação são distribuídos no Brasil (tabela 1). Dos 21 programas recomendados, 71,4\% têm o termo educação física em sua denominação; a expressão ciências do movimento humano é utilizada por $14,3 \%$ dos programas. Três instituições utilizam termos distintos e únicos: ciências de atividade física, ciências da motricidade e ciências do esporte. A obtenção de mestrado e doutorado é possivel em 47,6\% dos programas; nos demais, só é possivel obter o grau de mestre. 
Tabela 1 - Lista dos programas de pós-graduação recomendados pela CAPES com seus respectivos conceitos para os níveis de mestrado e doutorado.

\begin{tabular}{|c|c|c|c|c|}
\hline Denominação dos programas de pós-graduação & Universidade & Estado & M & D \\
\hline Ciências da Atividade Física & UNIVERSO & RJ & 3 & - \\
\hline Ciências da Motricidade & UNESP/RC & SP & 5 & 5 \\
\hline Ciências do Esporte & UFMG & MG & 4 & 4 \\
\hline Ciências do Movimento Humano & UFRGS & RS & 5 & 5 \\
\hline Ciências do Movimento Humano & UDESC & SC & 4 & 4 \\
\hline Ciências do Movimento Humano & UNICSUL & SP & 3 & - \\
\hline Educação Física & UnB & DF & 3 & - \\
\hline Educação Física & UCB & DF & 4 & 4 \\
\hline Educação Física & UFES & ES & 3 & - \\
\hline Educação Física & UFV/UFJF & $M G$ & 3 & - \\
\hline Educação Física & UFPR & PR & 4 & 4 \\
\hline Educação Física & UFRJ & RJ & 3 & - \\
\hline Educação Física & UGF & RJ & 5 & 5 \\
\hline Educação Física & UFPEL & RS & 3 & - \\
\hline Educação Física & UFSC & SC & 5 & 5 \\
\hline Educação Física & USP & SP & 6 & 6 \\
\hline Educação Física & UNICAMP & SP & 4 & 4 \\
\hline Educação Física & UNIMEP & SP & 3 & - \\
\hline Educação Física & USJT & SP & 4 & - \\
\hline Educação Física & FESP/UPE & PE & 3 & - \\
\hline Educação Física & UEL/UEM & PR & 3 & - \\
\hline
\end{tabular}

$\mathrm{M}=$ Conceito do mestrado; $\mathrm{D}=$ Conceito do doutorado. Sobre siglas, ver 0 apêndice $\mathrm{A}$.

(Fonte: $\quad<$ http://www.capes.gov.br/avaliacao/cursos-recomendados-e-reconhecidos>. Acesso em: out. 2009).

Os programas de pós-graduação em educação física concentram-se nas regiões Sul e Sudeste (gráfico1). A obtenção de títulos de doutorado só é possível nessas instituições; assim, a maioria dos acadêmicos com doutorado está concentrada em tais regiões. Um dos desafios para o sistema nacional de pós-graduação é aumentar a oportunidade de programas de qualidade fora do eixo Sul/Sudeste. Atualmente, a região Norte não tem um programa de pós-graduação em educação física, e a região Nordeste tem apenas um, sendo este em nível de mestrado.
Gráfico 1 - Distribuição dos programas por nível (mestrado ou mestrado e doutorado) e região do Brasil.

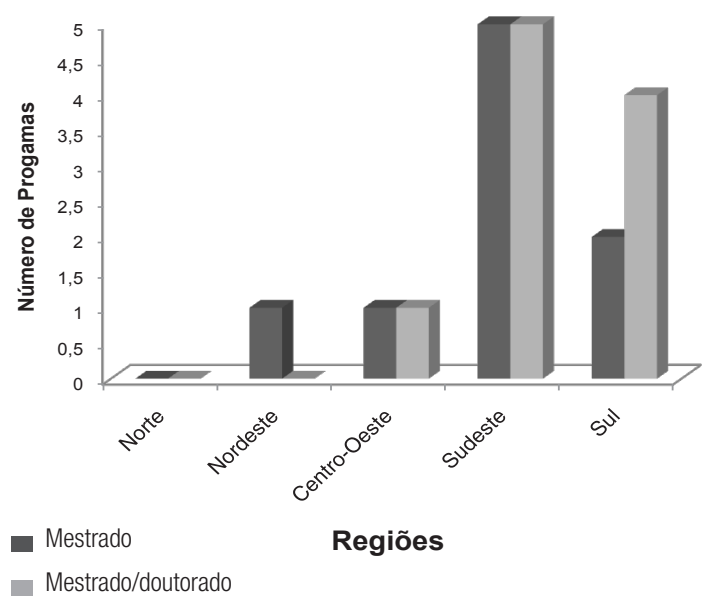

(Fonte: <http://www.capes.gov.br/avaliacao/cursos-recomendados-ereconhecidos>. Acesso em: out. 2009). 
Há uma concentração de programas (47,6\%) com conceito 3 (gráfico 2). 0 número de programas cai drasticamente à medida que o conceito aumenta, havendo um único programa com conceito 6 (4,7\%). A distribuição enviesada sugere que mais de um terço da área ainda não está consolidada. Essa distribuição pode ser entendida mais como resultado da política científica no país - particularmente em relação à grande área da saúde na CAPES - do que como um quadro da qualidade das atividades de ensino e pesquisa atualmente praticadas na maioria dos programas. Tal política tem induzido todas as instituições de ensino superior a seguirem um caminho para se tornarem internacionais. Aqueles que advogam a favor dessa política, fazem-no com base no rigor acadêmico. Entretanto, a distribuição dos conceitos reflete a aplicação de critérios com pouca consideração pela diversidade do campo. A consequência é o favorecimento de determinadas subáreas, em sua maioria fundamentadas nas ciências biológicas, em detrimento de outras, tais como aquelas orientadas para investigações de natureza educacional e sociocultural.

Gráfico 2 - Distribuição de programas por conceitos.

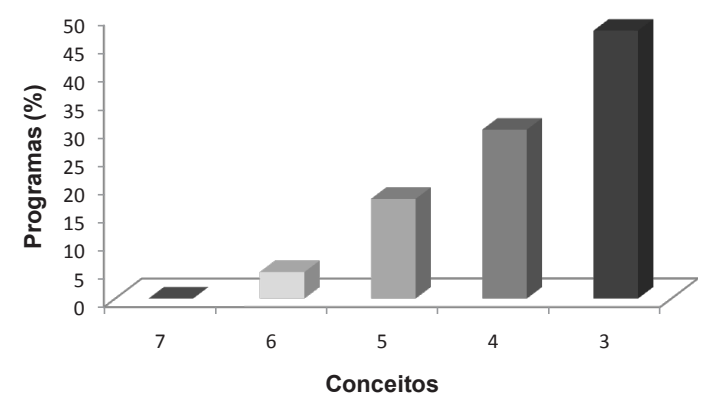

(Fonte: <http://www.capes.gov.br/avaliacao/cursos-recomendados-ereconhecidos>. Acesso em: out. 2009).
A distribuição dos conceitos por região do país oferece novas evidências de uma política científica centralizadora para a área (gráfico 3). Os programas com conceito 3 predominam em todas as regiões, enquanto os programas com conceitos 4,5 e 6 concentram-se nas regiões Sul e Sudeste. 0 único programa de pós-graduação na região Nordeste tem conceito 3.

Gráfico 3 - Distribuição dos programas por conceito por região do Brasil

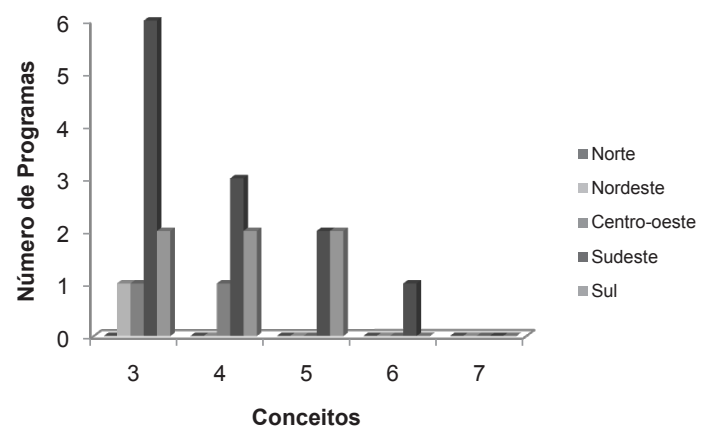

(Fonte: <http://www.capes.gov.br/avaliacao/cursos-recomendados-ereconhecidos > . Acesso em: out. 2009).

\section{A orientação acadêmica dos programas de pós-graduação em educação física}

A orientação acadêmica dos programas foi identificada pelo levantamento da dimensão do corpo docente e pelo número de linhas e projetos de pesquisa relativos a cada subárea: biodinâmica, sociocultural e pedagógica. 0 corpo docente corresponde aos professores permanentes (os envolvidos com atividades de pesquisa, de ensino - graduação e pós-graduação - e de orientação vinculadas a uma ou mais áreas de concentração) indicados pela instituição em seu relatório à CAPES. Na defınição da orientação acadêmica do docente, considerou-se a área em 
que seu doutorado foi obtido, juntamente com as linhas e projetos de pesquisa pelos quais é responsável (Gráfico 4).

Gráfico 4-Distribuição de docentes nas áreas de biodinâmica, sociocultural e pedagógica.

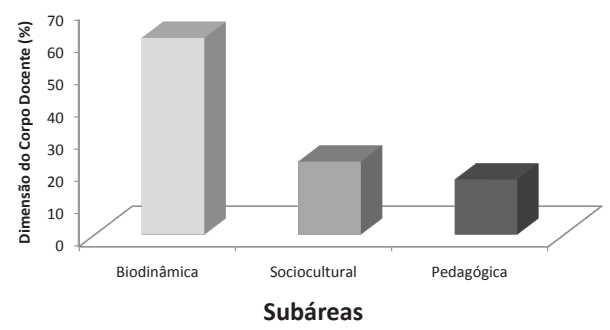

Fonte: <http://www.capes.gov.br/avaliacao/cursos-recomendados-ereconhecidos $>$. Acesso em: out. 2009).

Havia 293 docentes permanentes trabalhando em programas de pós-graduação no campo da educação física, de acordo com o levantamento de 2006. A maioria deles atua na subárea biodinâmica (60,7\% do total de docentes). Os demais docentes distribuem-se entre as subáreas sociocultural (22,52\%) e pedagógica (ao redor de 17\%). Para fazer parte do programa, o docente deve atender aos critérios estabelecidos pela comissão do próprio programa, a qual, em geral, segue os critérios praticados pela CAPES para recomendar programas. Assim, a distribuição das subáreas sociocultural e pedagógica resulta de uma combinação de fatores: refere-se à predominância da orientação biodinâmica dos programas e corresponde aos critérios de recomendação praticados pelos programas.

As linhas de pesquisa são consideradas ótimos indicadores para a orientação acadêmica dos programas. Elas caracterizam não só os temas e problemas específicos em que o pesquisador se envolve, como também as bases teóricas e metodológicas que ele elege como centrais à sua atividade. Assim, é possível a identificação da orientação acadêmica em termos das ciências naturais ou humanas e sociais. Cada linha de pesquisa tem, em geral, dois ou mais docentes envolvidos, e compreende um conjunto de projetos de pesquisa que comungam a base teórica, o nível de análise, os métodos e as técnicas. Novamente, a hegemonia da biodinâmica é marcante. De um total de 135 linhas de pesquisa identificadas em todos os programas, 50\% delas estão vinculadas à biodinâmica (gráfico 5). A subárea sociocultural tem 33\% do total de linhas de pesquisa, enquanto a subárea pedagógica tem $17 \%$.

Figura 5 - Distribuição de linhas de pesquisa pelas subáreas biodinâmica, sociocultural e pedagógica.

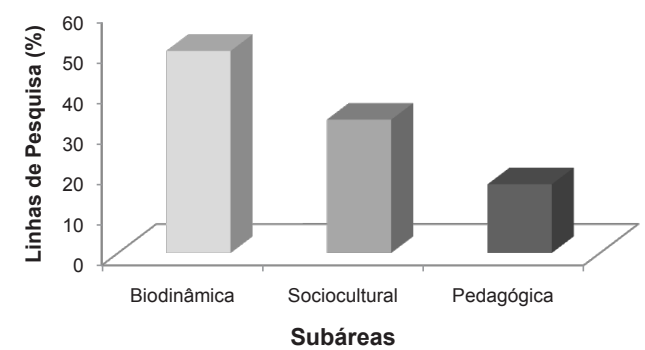

(Fonte: <http://www.capes.gov.br/avaliacao/cursos-recomendados-ereconhecidos $>$. Acesso em: out. 2009).

Os projetos de pesquisa são mais específicos que as linhas de pesquisa, já que eles se referem a problemas, questões e hipóteses particulares. Em geral, cada membro do corpo docente pode ter dois ou mais projetos de pesquisa sob sua responsabilidade. É nesse nível que a maioria dos pós-graduandos estão engajados na condução de pesquisas sob a supervisão de seus orientadores. Dos 860 projetos de pesquisa cadastrados em todos os programas da área, 67,4\% são da subárea biodinâmica.

Figura 6- Distribuição de projetos de pesquisa pelas subáreas biodinâmica, sociocultural e pedagógica

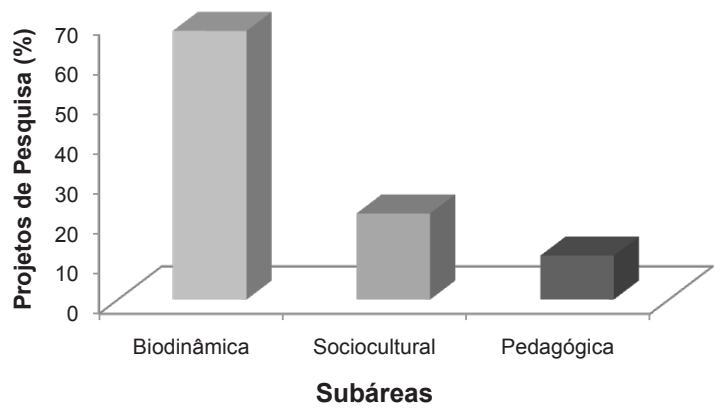

(Fonte: <http://www.capes.gov.br/avaliacao/cursos-recomendados-ereconhecidos>. Acesso em: out. 2009). 
Juntas, as subáreas sociocultural e pedagógica têm pouco mais de 30\% do total de projetos de pesquisa. Apesar de algumas diferenças na forma como os projetos foram concebidos em cada subárea, esses dados confirmam a tendência de que a biodinâmica é hegemônica na pós-graduação. Embora a educação física seja diretamente relacionada à intervenção - que é pedagógica, em essência -, ironicamente, projetos de pesquisa na subárea pedagógica correspondem a cerca de 10\% do total do número de projetos.

Doze programas têm a biodinâmica como subárea predominante, o que corresponde a 57\% do total de programas da área (tabela 2). 0 conceito de um programa demonstra estar associado à sua orientação. Por exemplo, com uma exceção, a biodinâmica predomina em programas com conceitos 5 e $6(80 \%$ do total). Entre os programas com conceito 4, 66,7\% apresentam a biodinâmica em suas subáreas. Nos programas com conceito 3, as subáreas sociocultural e pedagógica são predominantes (mais de 60\%). Em geral, os programas com os melhores conceitos são aqueles em que a biodinâmica é predominante. Ao mesmo tempo, à medida que o conceito diminui, há uma diminuição correspondente da presença da biodinâmica nos programas.

Tabela 2 - Relação entre a presença da biodinâmica no programa e seu conceito.

\begin{tabular}{|c|c|c|}
\hline UNIVERSIDADE & BIODINÂMICA (\%) & Conceito \\
\hline USP & 72,7 & 6 \\
\hline UFSC & 62,5 & 5 \\
\hline UGF & 25 & 5 \\
\hline UFRGS & 66,67 & 5 \\
\hline UNESP/RC & 55,56 & 5 \\
\hline UDESC & 66,67 & 4 \\
\hline UCB & 66,67 & 4 \\
\hline UFPR & 75 & 4 \\
\hline UNICAMP & 50 & 4 \\
\hline UFMG & 75 & 4 \\
\hline USJT & 25 & 4 \\
\hline UNIVERSO & 42,8 & 3 \\
\hline UNICSUL & 100 & 3 \\
\hline UnB & 66,67 & 3 \\
\hline UFES & 0 & 3 \\
\hline UFV/UFJF & 50 & 3 \\
\hline UFRJ & 100 & 3 \\
\hline UFPel & 50 & 3 \\
\hline UNIMEP & 40 & 3 \\
\hline FESP/UPE & 50 & 3 \\
\hline UEL/UEM & 66,67 & 3 \\
\hline
\end{tabular}

(Fonte: <http://www.capes.gov.br/avaliacao/cursos-recomendados-e-reconhecidos>. Acesso em: out. 2009). 
No conjunto, os dados destacam a hegemonia da biodinâmica nos programas de pós-graduação em educação física. Essa tendência é também observada para os novos programas recomendados nos últimos três anos. A maioria deles confirma a ampla presença da biodinâmica, o que muitos entendem ser condição para que um programa seja recomendado (tabela 3). Desses programas, 50\% têm pelo menos só essa subárea; a outra metade tem predominância dessa subárea, com apenas uma exceção.

Tabela 3 - Relação entre a presença da biodinâmica nos programas novos e seu conceito.

\begin{tabular}{c:c:c}
\hline UnIVERSIDADE & BIODINÂMICA (\%) & ConCEITO \\
\hline UNICSUL & 100 & 3 \\
\hline UnB & 66,67 & 3 \\
\hline UFES & 0 & 3 \\
\hline UFV/UFJF & 50 & 3 \\
\hline UFRJ & 100 & 3 \\
\hline UFPel & 50 & 3 \\
\hline FESP/UPE & 50 & 3 \\
\hline UEL & 66,67 & 3 \\
\hline
\end{tabular}

(Fonte: <http://www.capes.gov.br/avaliacao/cursos-recomendados-ereconhecidos>. Acesso em: out. 2009).

\section{Consideraçōes finais}

0 impacto que os recursos humanos na educação superior, na ciência e na tecnologia têm na sociedade tornou os programas de pós-graduação um lugar cobiçado para se estar na universidade. Não é surpreendente que universidades públicas e privadas tenham devotado atenção especial a seus programas de pós-graduação e esperem ansiosas pela divulgação dos resultados da avaliação trienal conduzida pela CAPES. Assim, as propostas dos programas de pós-graduação podem ser vistas como uma resposta que as instituições dão às políticas de Estado. As propostas tam- bém resultam de lutas internas e externas pela hegemonia acadêmica. Programas brasileiros de pós-graduação em educação física estão constituídos orientados pela concepção anglo-saxã. Assim, há algumas aproximações entre a educação física brasileira e a norte-americana.

Nos Estados Unidos, a matriz epistemológica da cinesiologia é disciplinar. No Brasil, a situação é ambígua, uma vez que educação físi$c a$ é o termo preferido por instituições de ensino superior e está presente amplamente na denominação dos programas de pós-graduação. Todavia, as áreas de concentração desses programas revelam uma matriz disciplinar com três subáreas - biodinâmica, sociocultural e pedagógica -, havendo uma hegemonia da primeira.

Alguns acadêmicos norte-americanos têm apontado as dificuldades em integrar e mesmo incluir as subáreas com orientação sociocultural e pedagógica dentro do arcabouço epistemológico no qual a cinesiologia é fundada (VERTINSKY, 2009). No Brasil, um processo similar ocorre em relção à coexistência problemática das subáreas sociocultural e pedagógica com a biodinâmica. Essa dificuldade expõe o fato de que muitos acadêmicos brasileiros têm uma visão limitada dos interesses e necessidades da sociedade e do papel da educação física em atendê-los.

Jane Clark (2008) argumenta que a cinesiologia nos Estados Unidos vive um momento auspicioso, haja vista a área ter sido finalmente aceita para avaliação pelo Conselho Nacional de Pesquisa dos Estados Unidos (THOMAS et al., 2007). Há algumas similaridades preocupantes entre a cinesiologia dos Estados Unidos e a subárea biodinâmica na educação física brasileira. Por exemplo, Jerry Thomas e Thomas Reeve (2006) relatam que alguns departamentos norte-americanos estão engajados em substituir a cinesiologia por algo como biologia ou fisiologia integrativa. Investigações que privilegiam questões socioculturais e pedagógicas têm perdido espaço no campo acadêmico, seja ele denominado de cinesiologia ou de educação física. Patricia Vertinsky (2009) relatou que, no 
Canadá, muitos acadêmicos da subárea pedagógica estão migrando para outras áreas, como a educação, em busca de ter seu trabalho mais reconhecido e valorizado. David Andrews (2008) também argumenta que, embora muitos departamentos ainda mantenham disciplinas socioculturais em sua estrutura acadêmica, o corpo docente é dominado por aqueles que trabalham nas disciplinas de orientação biológica.

Vertinsky (2009) assinala que a crescente presença da cinesiologia cria um movimento para privilegiar a pesquisa quantitativa com ênfase em fenômenos naturais e na identificação dos mecanismos subjacentes a eles. Em contraposição, as preocupações com fenômenos sociais, pesquisas qualitativas e estudos interpretativos têm sido postas de lado. 0 que está sendo apontado por Vertinsky não é novo. Há 15 anos, Linda Bain (1995) já clamava pela necessidade de o campo considerar outras formas de conhecimento e de produção de conhecimento.

No Brasil, os docentes afetos aos temas das subáreas socioculturais e pedagógicas perdem espaço nos programas de pós-graduação. Suas produções científicas são desqualificadas e, no cotidiano do trabalho na universidade, enfrentam as pressões das gestões e de órgãos de fomento que privilegiam e investem em pesquisa baseada em um modelo de ciência que não considera a diversidade e a singularidade da natureza dos objetos de investigação. Há um abismo crescente entre as prioridades das universidades e os dilemas da sociedade que caracterizam a necessidade de informação, conhecimento e intervenção responsáveis e adequados. A condução desse processo de exclusão gradual a que os docentes das subáreas pedagógicas e socioculturais têm sido submetidos tem, no sistema de avaliação da pós-graduação, um de seus grandes vilões (BETTI et al., 2004).

Um processo similar ocorre na América do Norte. Por exemplo, os critérios de avaliação desenvolvidos pelo comitê apontado pela Aliança Americana de Cinesiologia e Educação Física (AAKPE) estabeleceram que um artigo em periódico vale de 15\% a 20\% mais do que um livro (THOMAS; REEVE, 2006; THOMAS et al., 2007). Isso mostra como os livros ainda são desvalorizados pelos comitês de avaliação, apesar de serem um dos principais veículos de divulgação científica da produção das subáreas sociocultural e pedagógica. Vertinsky (2009) relata sua própria experiência no Canadá com sua produção no formato de livro: livro e capítulo de livro valiam 20\%, 30\% e até 40\% menos do que uma produção em artigo. A desvalorização dos livros fez com que ela mudasse o tipo de pesquisa e de metodologia que usualmente utilizava, levando-a a buscar resultados quantitativos que teriam mais chances de serem publicados em artigos. Para atender a um critério de avaliação, ela viu-se obrigada a sacrificar sua competência intelectual.

Richard Tinning (2008) destaca que a educação física tem suas origens na pedagogia como campo de investigação; entretanto, apenas recentemente sua presença como área é notada nos departamentos americanos, mas, mesmo assim, com problemas de ordem conceitual e de identidade. No Brasil, a subárea pedagógica é a menor nos programas de pós-graduação, o que contribui para o aumento da distância entre o que se pesquisa nas universidades e os interesses e necessidades da sociedade.

Os conhecimentos produzidos pela cinesiologia em geral e pela biodinâmica em particular têm grande potencial de generalização, mas as aplicações na resolução de problemas e no desenvolvimento de bens e serviços requerem um investimento na pesquisa orientada aos dilemas que as populações enfrentam. Os estudos pedagógicos são delineados para responder a tal demanda. Restringir essa produção com base em uma política científica que se justifica por meio de critérios que valorizam a produtividade quantitativa, em detrimento do impacto e da relevância social da produção científica, implica também abdicar das pesquisas que legitimam, acadêmica e profissionalmente, a educação física. Judith Rink (2007) destaca que essa política é refletida na preparação profissional, 
em que há um desequilíbrio entre as disciplinas pedagógicas e aquelas dedicadas à compreensão dos mecanismos do movimento humano. Estudantes de graduação no Brasil e nos Estados Unidos sabem cada vez mais sobre as bases moleculares da contração muscular e menos sobre como planejar um currículo e conduzir uma aula. Esse desequilíbrio reflete o investimento que se faz nas pesquisas em biodinâmica, em detrimento das pesquisas pedagógicas.

Qual é o caminho a seguir? Andrews (2008) e Vertinsky (2009) concordam que a cinesiologia só teria a ganhar acolhendo a área sociocultural. Andrews propôs a constituição de uma área de estudos da cultura física como aglutinadora de vários docentes, cujas pesquisas seriam orientadas pelas ciências sociais e humanas. Essa área consistiria em uma síntese de influências empíricas, teóricas e metodológicas de várias subdisciplinas isoladas (entre as quais estariam a sociologia e a história do esporte e da atividade física). Vertinsky (2009) considera que a área sociocultural poderia trazer os elementos necessários para a constituição de um campo verdadeiramente interdisciplinar. A cinesiologia, apesar de apresentar-se como interdisciplinar, consiste, de fato, no conjunto de disciplinas isoladas.

$\mathrm{Na}$ perspectiva de Rink (2007), deveria haver um esforço para transformar a atual graduação, orientado-a para aqueles que escolheram atuar profissionalmente com educação física dentro e fora das escolas. Rink fala sobre a necessidade de aproximar os estudos pedagógicos da cinesiologia a partir de um compromisso entre os acadêmicos das duas subáreas; os da cinesiologia fariam um esforço para realizar pesquisas a partir de questões que emanam da prática. Isso poderia resultar em um conhecimento básico que teria maior interesse para o futuro profissional. Ao mesmo tempo, os pesquisadores da subárea pedagógica estariam envolvidos em identificar qual conhecimento básico sobre o movimento humano seria mais relevante para enfrentar os problemas da prática.
Os artigos de Andrews (2008) e Vertinsky (2009), assim como os dados apresentados aqui sobre a realidade brasileira, mostram que a hegemonia da biodinâmica não é um fato isolado. Ela guarda relação com uma tendência mundial na qual as universidades se voltam para as chamadas tecnociências (ARAÚJO, 1998), ciências a serviço de interesses econômicos e políticos que passam ao largo do compromisso com valores universais como justiça, igualdade, liberdade de expressão e verdade (SAID, 2005). Como lembra Vertinsky (2009), no embate entre as subáreas encontramos os ecos da polarização entre ciências naturais e humanidades, descrita por Charles Snow (1995) há mais de 50 anos. Vertinsky (2009) sugere que há terreno para uma aproximação, recorrendo à proposição de Stephen Gould sobre o tema. Gould (2003) fala sobre uma conciliação, considerando que as diferenças entre as ciências naturais e as humanidades são benéficas para ambas e não devem ser eliminadas. 0 autor argumenta que não importa o que façamos, a ciência segue um caminho diferente das humanidades e vice-versa, e são essas diferenças que tornam importante a aproximação entre elas. Apesar das esperanças de Vertinsky (2009), somos pessimistas a respeito da conciliação nos termos de Gould. A lógica da cinesiologia é mais suscetível a outra proposta de aproximação: a de consiliência, de Edward Wilson (1998). Seu argumento é em favor de uma unidade da ciência e do conhecimento; entretanto, tal unidade seria operada a partir do paradigma das ciências naturais, implicando a redução das humanidades à ciência.

Tinning (2008) propõe rever todas as subdisciplinas da cinesiologia sob a ótica de uma pedagogia crítica, como forma de refletir e questionar os modos de (re)produção do conhecimento em cada uma delas. Esse seria um modo de reestruturar as subdisciplinas e criar um território comum entre elas. A proposta de inning é ousada e sua implementação implicaria uma crítica do próprio modelo de ciência que sustenta a cinesiologia. 
Em que pese a necessidade de aproximação entre saberes e práticas apontada por Snow e Gould (2003), a problemática atual vai além de uma possível conciliação entre subáreas ou subdisciplinas. 0 esforço de aproximação e promoção de uma coexistência pacífica entre elas toca diferentes questões, sobretudo a luta pelo poder que se trava entre os muros da universidade. A hegemonia de determinados grupos significa: (a) controle sobre os critérios de concessão de auxílios e recursos financeiros para a pesquisa; (b) obtenção das maiores fatias desse bolo de recursos; e, (c) controle na admissão de pessoal para integrar o corpo acadêmico dos departamentos. Tudo isso serve para manter o status quo dos que estão no poder nas instituições universitárias. 0 que estamos vivendo na universidade é a reprodução de um processo que transcende seus muros. A universidade, a cada dia e com maior velocidade, transforma-se em uma instituição comprometida e redefinida de acordo com a lógica e as leis do mercado, com a organização eficaz e o tecnicismo produtivo (SILVA, 2006). Tudo isso ocorre à custa de recursos públicos $\mathrm{e}$ na contramão do movimento em defesa de um ensino universitário decente e responsável.

\section{Universidades brasileiras com programas de pós-graduação em edu- cação física e seus respectivos estados}

UNIVERSO: Universidade Salgado Filho; RJ: Rio de Janeiro

UNESP/RC: Universidade Estadual Paulista “Julio de Mesquita Filho”, Campus Rio Claro; SP: São Paulo

UFMG: Universidade Federal de Minas Gerais; MG: Minas Gerais

UFRGS: Universidade Federal do Rio Grande do Sul; RS: Rio Grande do Sul

UFRJ: Universidade Federal do Rio de Janeiro; RJ: Rio de Janeiro

UDESC: Universidade Estadual do Estado de Santa Catarina; SC: Santa Catarina

UNICSUL: Universidade Cruzeiro do Sul; SP: São Paulo

UnB: Universidade de Brasília; DF: Distrito Federal

UCB: Universidade Católica de Brasília; DF: Distrito Federal

UFES: Universidade Federal do Espírito Santo; ES: Espírito Santo

UFV/UFJF: Universidade Federal de Viçosa; MG: Minas Gerais

UFPR: Universidade Federal do Paraná; PR: Paraná

UGF: Universidade Gama Filho; RJ: Rio de Janeiro

UFPEL: Universidade Federal de Pelotas; RS: Rio Grande do Sul

UFSC: Universidade Federal de Santa Catarina; SC: Santa Catarina

USP: Universidade de São Paulo; SP: São Paulo

UNICAMP: Universidade Estadual de Campinas; SP: São Paulo

UNIMEP: Universidade Metodista de Piracicaba; SP: São Paulo

USJT: Universidade São Judas Tadeu; SP: São Paulo

FESP/UPE: Fundação do Estado de Pernambuco / Universidade de Pernambuco; PE: Pernambuco

UEL/UEM: Universidade Estadual de Londrina; PR: Paraná 


\section{Referências}

ABERNETHY, B. et al. The biophysical foundations of human movement. Champaign: Human Kinetics, 1996.

AMADIO, A.; BARBANTI, V. (Orgs.). Biodinâmica do movimento humano e suas relações interdisciplinares. São Paulo: Estação Liberdade, 2000.

ANDREWS, D. Kinesiology's inconvenient truth and the physical cultural studies imperative. Quest, v. 60, p. 45-62, 2008.

ARAÚJO, H. (Org.). Tecnociência e cultura: ensaios sobre o tempo presente. São Paulo: Estação Liberdade, 1998.

BAIN, L. The fourth Delphine Hanna lecture: mindfulness and subjective knowledge. Quest, v. 47, p. 253-283, 1995.

BETTI, M. Por uma teoria da prática. Motus Corporis, v. 3, p. 73-127, 1996.

BETTI, M. et al. A avaliação da educação física em debate: implicações para a subárea pedagógica e sociocultural. Revista Brasileira de Pós-Graduação, v. 1, p. 183-194, 2004.

BRACHT, V. Mas afinal o que estamos perguntando com a pergunta "o que é educação física". Movimento, v. 2, n. 2, p. 1-8, 1995.

Educação física e ciência: cenas de um casamento (in)feliz. Campinas: Autores Associados, 2003.

Por uma política científica para a educação física com ênfase na pós-graduação. In: FÓRUM NACIONAL PERMANENTE

DE PÓS-GRADUAÇÃO EM EDUCAÇÃO FÍSICA, 2006, Campinas. Disponível em: <http://www.cbce.org.br/br/acontece/materia. asp?id=312>.

. 0 CBCE e a pós-graduação stricto sensu da educação física brasileira. In: CARVALHO, Y.; LINHALES, M. (Orgs.). Política científica e produção do conhecimento em educação física. Goiânia: Colégio Brasileiro de Ciências do Esporte, 2007. p.7385.

CARVALHO, Y. M.; MANOEL, E. J. Para além dos indicadores de avaliação da produção intelectual na grande área da saúde. Movimento, v. 12, n. 3, p. 193-225, 2006.

. 0 livro como indicador de produção intelectual na grande área da saúde. Revista Brasileira de Ciências do Esporte, v. 29, p. 61-73, 2007.

CARVALHO, Y. M. et al. O livro na pós-graduação: uma metodologia para avaliação do livro. Revista Brasileira de Pós-Graduação, v. 5, n. 10, p. 226-249, 2008.

CLARK, J. Kinesiology in the 21st century: a preface. Quest, v. 60, p. 1-2, 2008.

CORYN, C. The use and abuse of citations as indicators of research quality. Journal of Multidisciplinary Evaluation, v. 4, p. 115-121, 2005.

DANTAS, F. Responsabilidade social e pós-graduação no Brasil: idéias para (avali)ação. Revista Brasileira de Pós-Graduação, v. 1, n. 2, p. 160-172, 2004

FENSTERSEIFER, P. A contribuição da filosofia para a área de educação física e/ou ciências do esporte. Revista Brasileira de Ciências do Esporte, v. 17, p. 167-171, 1996.

FERREIRA, M. M.; MOREIRA, R. L. (Orgs.). CAPES 50 anos: depoimentos ao CPDOC/FGV. Brasília: FGV/CPDOC/CAPES, 2002.

GARFIELD, E. How to use citation analysis for faculty evaluations and when it is relevant. Part 1. Essays of an Information Scientist, v. 6, n. 44, p. 354-362, 1983. 
. Dispelling a few common myths about journal citation impacts. The Scientist, v. 11, n. 3, p. 11, 1994.

GAYA, A. Mas afinal o que é educação física? Movimento, v. 1, n. 1, p. I-IV, 1994.

GOULD, S. J. The hedgehog, the fox and the magister's pox: mending the gap between science and the humanities. New York: Harmony Books, 2003.

HENRY, F. M. Physical education: an academic discipline. Journal of Health, Physical Education \& Recreation, v. 35, p. 32-33, 1964.

HOPKINS, W. Journal impact factors in sport and exercise science, 1999-2001. Sportscience, v. 5, n. 3. 2001. Disponível em: <sportsci.org/jour/0103/wgh.htm>. Acesso em: 20 set. 2005.

KOKUBUN, E. Pós-graduação em educação física no Brasil: indicadores objetivos dos desafios e das perspectivas. Revista Brasileira de Ciências do Esporte, v. 24, p. 9-26, 2003.

KRETCHEMAR, R. S. Practical philosophy of sport. Champaign: Human Kinetics, 1994.

LOVISOLO, H. Mas, afinal, o que é educação física?: a favor da mediação contra os radicalismos. Movimento, v. 2, n. 2, p. 18-24, 1995.

Hegemonia e legitimidade nas ciências dos esportes. Motus Corporis, v. 3, n. 2, p. 51-72, 1996.

. Sobre a pós-graduação em educação física. In: NETO, A. F. (Org.). Leituras da natureza científica do Colégio Brasileiro de Ciências do Esporte. Campinas: Autores Associados, 2005. p.71-90

MACHADO, A.; LOURENÇO, 0.; SILVA, F. Facts, concepts, and theories: the shape of psychology's epistemic triangle. Behavior and Philosophy, v. 28, p. 1-40, 2000.

NEWELL, K. Kinesiology: the label for the study of physical activity in higher education. Quest, v. 42, p. 279-296, 1990.

NOGUEIRA, M. A.; AGUIAR, A. M. S.; RAMOS, V. C. C. Fronteiras desafiadas: a internacionalização das experiências escolares. Educação \& Sociedade, v. 29, n. 103, p. 355-376, 2008.

PARK, R. A long and productive career: Franklin M. Henry - scientist, mentor, pioneer. Research Quarterly for Exercise and Sport, v. 65, p. 295-307, 1994.

RARICK, L. The domain of physical education. Quest, v. 9, p. 49-52, 1967.

RINK, J. What knowledge is of most worth? Perspectives on kinesiology from pedagogy. Quest, v. 59, p. 100-110, 2007.

RODRIGUES, L. O. Publicar mais ou melhor? 0 tamanduá olímpico. Revista Brasileira de Ciências do Esporte, v. 29, p. 35-48, 2007.

SAID, E. Representações do intelectual. São Paulo: Companhia das Letras, 2005.

SANTIN, S. A respeito de comentários. Movimento, v. 2, n. 2, p. 9-14, 1995.

SILVA, F. L.. Universidade: a idéia e a história. In: STEINER, J.; MALNIC, G. (Orgs.). Ensino superior: conceito \& dinâmica. São Paulo: Edusp, 2006. p.285-295.

SNOW, C. P. As duas culturas e uma segunda leitura. São Paulo: Edusp, 1995.

SOARES, C. Imagens da educação no corpo: estudo a partir da ginástica francesa no século XIX. Campinas: Autores Associados, 1998. 
TAFFAREL, C.; ESCOBAR, M. Mas, afinal, o que é Educação Física?: um exemplo do simplismo intelectual. Movimento, v. 1, n. 1, p. 5-8, 1994.

TANI, G. Cinesiologia, educação física e esporte: a ordem emanante do caos na estrutura acadêmica. Motus Corporis, v. 3, p. 9-50, 1996.

THOMAS, J.; REEVE, T. G. A review and evaluation of doctoral programs 2000- 2004 by the American Academy of Kinesiology and Physical Education. Quest, v. 58, p. 176-196, 2006.

THOMAS, J. et al. The academy promotes, unifies, and evaluate doctoral education in kinesiology. Quest, v. 59, p. 174-194, 2007.

TINNING, R. Pedagogy, sport pedagogy and the field of kinesiology. Quest, v. 60, p. 405-424, 2008.

VERTINSKY, P. Mind the gap (or mending it): Qualitative research and interdisciplinarity in kinesiology. Quest, v. 61, p. 39-51, 2009.

WATERS, L. Inimigos da esperança: publicar, perecer e o eclipse da erudição. São Paulo: Editora da Unesp, 2006.

WILSON, E. Consilience: the unity of knowledge. New York: Alfred Knopf Inc., 1998.

Recebido em 21.04.10

Aprovado em 12.09.10

Edison de Jesus Manoel é professor titular da Universidade de São Paulo (2003); livre-docente em Pedagogia do Movimento Humano (USP, 1998); doutor em Psicologia (University of Sheffield, Grã Bretanha, 1993); mestre em Educação Física (USP, 1989); licenciado em Educação Física (USP, 1980).

Yara Maria de Carvalho é professora associada da Universidade de São Paulo; livre-docente em promoção da Saúde pela Faculdade de Saúde Pública da Universidade de São Paulo (2010); doutora em Saúde Coletiva pela Faculdade de Ciências Médicas da Universidade Estadual de Campinas (1999); pós-doutora pela Universidade La Sapienza de Roma, Itália (2003/2004); diretora científica do Colégio Brasileiro de Ciências do Esporte. E-mail: yaramc@usp.br. 